\title{
Atmospheric optical communication
}

\author{
Cardinal Warde \\ Massachusetts Institute of Technology \\ Department of Electrical Engineering and Computer Science \\ and the Center for Materials Science and Engineering \\ Cambridge, Massachusetts 02139
}

Fiber optic communication generally comes to mind first when one thinks or speaks of optical communication. In fact, the two terms are often used synonymously. Indeed, the fiber optic communication technology is maturing rapidly and it offers enormous advantages over unguided atmospheric optical propagation systems. However, there is a variety of applications for which fiber systems are impractical and for which atmospheric optical systems may be preferred. For example, short-haul, over-thehorizon links in areas where cable real estate rights are unobtainable, or where an optical broadcast mode is desired, or for spaceto-earth optical links.

The impressive advances that have been accomplished in the area of fiber optic communication have been fairly well documented in review papers, books, and special issues of journals devoted to this subject. In this issue, we present a limited review of the lesser known area of atmospheric optical communication.

The atmosphere is an optical propagation channel whose characteristics are constantly changing. The propagation characteristics of the clear turbulent atmosphere (randomly fluctuating index of refraction medium) are fairly well understood, and relatively simple atmospheric optical communication systems can be designed to operate efficiently through clear-air turbulence. However, as the aerosol (smoke, haze, fog, clouds, etc.) content of the atmosphere increases, the effects of multiple scattering, viz., loss of coherence, multipath spread (time dispersion), Doppler spread (frequency dispersion), and angular spread (spatial frequency dispersion) can severely limit the performance of atmospheric optical systems.

The propagation characteristics of the low-visibility atmosphere are, therefore, of extreme importance to the design and utilization of all-weather communication systems. Unfortunately, multiple-scatter theory does not yet provide an explicit determination of the aforementioned spread parameters that characterize the low-visibility atmospheric channel. Multiple scatter theory, therefore, continues to be an area of active research. The first paper, by A. Ishimaru, is a review of the present state of the art in the theory of optical propagation through turbulent and turbid atmospheres, and as the author so well points out, much work remains to be done in this area.

Experimental methods of characterizing the atmosphere as a communication channel have been of great value in aiding the design of practical communication systems, as well as for providing guidance as to realistic approximations that could simplify the multiple-scatter theoretical calculations. The second paper, by $G$. C. Mooradian, summarizes a series of optical propagation measurements that were taken with actual surface and subsurface optical communication systems operating in a marine environment. (A second channel characterization paper based on experimental measurements, authored by W. S. Ross et al., of Massachusetts Institute of Technology, could not be prepared on time for this special issue and will appear in a later issue.)

In severe low-visibility weather, atmospheric optical communication systems will undoubtedly suffer operating range limitations and outage periods. However, by suitably processing and utilizing the scattered component of the received light, it should be possible to improve communication performance under these conditions. The third paper, by J. H. Shapiro and C. Warde, takes a unified approach to the issues of atmospheric propagation, communication, and signal processing. In particular, it summarizes the multiple-forward-scatter propagation model, shows that background light suppression is the key to extended link operability under low-visibility conditions, and describes recent work on space-distributed phase compensation techniques for achieving background light suppression in line-of-sight links.

The papers presented here are only samples from the various research efforts in the field of atmospheric optical communication. Active research continues in the areas of novel receiver structures, receiver postprocessing schemes, frequency hopping systems, frequency modulation systems, and schemes for quantum communication. Several inexpensive systems using LEDs and lasers as sources have been demonstrated, and a few are commercially available. As technological advances improve the performance of atmospheric optical communication systems, they will find increasing application in the future. 